\title{
Comparators (Medicinal and non Medicinal) for Marketing Authorization, for Public Health, for Payers and at the European Level
}

\author{
Driss Berdaï ${ }^{1}$, Jean-Michel Hotton ${ }^{2}$, Philippe Lechat ${ }^{3}$ and participants of Round Table $n^{\circ} 1$ Giens XXV ${ }^{\star}$ \\ 1 CHU de Bordeaux, Bordeaux, France \\ 2 Pfizer, Paris, France \\ 3 Afssaps, Saint-Denis, France
}

Text received 29 march 2010; accepted 8 june 2010

\begin{abstract}
Keywords:
clinical trial; control group; comparator; clinical benefit; health fund; recommendation

Abstract - Drug evaluation is based on comparison. Thus, the choice of the comparator for any new treatment becomes a key issue, especially when there are great differences in medical practice and of use conditions of the comparators depending on the geographical zones and their evolution with time. The choice of the comparators must satisfy sometimes different expectations from the registration authorities and for insurance coverage. The universal comparator that allows answering all the clinical assessment questions does not exist. Placebo, when it can be used, remains a reference for the MA (marketing authorisation) application, but does not exclude the use of the reference drug available on the market and prescribed under optimal efficacy conditions. The reference treatment is sometimes a difficult choice due to the absence of validated therapeutic recommendations or if the recommendations vary depending on the countries. The expansion and international harmonization of prescription guidelines (clinical practice guidelines) would reinforce the robustness and efficiency of clinical research efforts with respect to the relevance of the comparison to reference treatments. This principle also applies to the use of a non-drug comparator when it has been recognized as the reference comparator in the treatment of the pathology in question. In as much as possible, the search for a consensus must also aim at defining in the clinical development recommendations significant thresholds for the size of evaluated effects. Optimization of the information made available after clinical trials could also be helped by the development of use of methodologies that allow assessing superiority on secondary criteria during a non-inferiority study on the main criterion. Finally, the development of early scientific consultations by the Haute Autorité de Santé (HAS, French Health Authority) would contribute to adapt phase III clinical trials better to questions concerning the assessment of the clinical added value of the medicinal products evaluated.
\end{abstract}

\section{Introduction}

The comparison exercise is the basis of the scientific assessment method in medicine, in particular within the framework of controlled clinical trials. ${ }^{[1]}$

This experimental process is made difficult by the diversity of medicinal and non-medicinal comparators that can be applied to similar clinical situations. However, this method is essential in order to provide pertinent answers to questions of optimal treatment of patients during the clinical development of drugs. Furthermore, the information provided by the clinical trials should allow preparing MA (Marketing Authorisation) applications, ${ }^{[2]}$ answer-

\footnotetext{
^ For a list of participants, see end of article.
}

ing questions concerning their coverage by health funds and during the fixing of their price, ${ }^{[3,4]}$ these regulatory steps being unavoidable prior to making the drugs available to the majority of patients who may benefit from them. ${ }^{[5]}$

\section{Identified constraints}

2. 1. The absence of consensus on the method of optimal treatment of patients and target diseases $(e . g$. absence of medical practice consensus)

Therapeutic progress rests in particular on the results made available after several thousands of new clinical trials started each 
year. ${ }^{[6]}$ Beyond the limits to extrapolate results observed in experimental situation to those attained in real life, ${ }^{[7]}$ the other difficulty encountered by clinicians is translating the results into operational data that may applied to everyday life. ${ }^{[8,9]}$ This difficulty is balanced by the results of the assessment of medicinal products by the competent authorities. The latter validates and makes available reference documents that have a real practical value (e.g. summary of product characteristics, opinions and summaries of opinions of the French Transparency Commission, etc.). This considerable effort on the systematic assessment and codification of usages has allowed major progress in the harmonization of practices with time.

However, professional practices remain variable for similar medical conditions. These differences may in particular be explained by the absence of valid medical practice recommendations (clinical practice guidelines) or by divergent recommendations between countries or institutions. Clinical practices that are regularly validated and updated would be more pertinent to consider when choosing a comparison group during the clinical trial of a new drug. ${ }^{[10,11]}$ One can thus understand the importance of the development of clinical practice guidelines, harmonized at an international or at least European level in order to optimize pivotal clinical trials.

\subsection{Geographic variability of reference medical practices}

A major international harmonization effort has been carried since numerous years in the drug assessment field, ${ }^{[12]}$ both by the availability of clinical development recommendations [at a European scale via the European Medicines Agency (EMA) and Committee for Medicinal Products for human use (CHMP), or international through the International Conference on Harmonisation (ICH) process] but also by mutualization of MA application assessment resulting in an increasing homogeneity of the summary of product characteristics within the European Union. However, only the most recent drugs have been subjected to such procedures. Furthermore, in clinical practice, the clinical development recommendations as well as the therapeutic treatment recommendations are established per pathologies and not per specific proprietary medicinal products. This results in a still excessive disparity in clinical uses of drugs depending on the countries, without necessarily having a medically valid explanation (e.g. different genetic profiles requiring an appropriate mode of administration). Furthermore, some guidelines recommend different therapeutic practices depending on the country even though the target diseases have similar clinical expressions (e.g. asthma).
2.3. Variations in conditions for use of reference treatments according to the country (formulations, dosages, etc.)

Apart from the considerations developed in the previous paragraph, the choice of a single comparator during a phase III pivotal clinical trial may be complicated by different methods of use for the same drug substance depending on the countries considered such as: pharmaceutical forms (solution, slow release formulation, etc.), posologies, chronologies of administration or routes of administration. This is in particular the case of comparators for which the MA may be different from one country to another within the European Union prior to the application of the centralized procedure.

\subsection{The evolution of practice with time (e.g. reference drug for which the MA is granted over the clinical development period of a drug targeting the same indication)}

In areas of high clinical research activity, pace of innovation may be such that the therapeutic reference may change over the clinical development period. Thus, a comparator that was valid at the time the protocol was elaborated may become obsolete at the time the marketing authorization application dossier is filed or at the time of the application for health insurance coverage.

2.5. Multiple information to be provided to many interested parties, including international authorities, based on data from a single clinical development and a limited number of phase III studies

The information expected at the end of drugs' clinical trials is diverse. ${ }^{[13]}$ Depending on the questions asked, the methodological choices and the constitution of the comparator groups vary, ${ }^{[14]}$ in particular:

- the use of placebo ${ }^{[15]}$ (when it can be used) is usually required by the competent authorities to evaluate a marketing authorization application;

- the use of a reference treatment (medicinal or not) is required for the assessment of the relative benefits and risks concerning the new treatment evaluated and as such will be strongly recommended for a MA application and as support for an application for coverage by health insurance organizations. ${ }^{[16]}$

- other methodologies or specific analyses may be necessary in order to satisfy the efficiency assessment requirements (cost-effectiveness) or the budgetary impact (budget impact analysis). 
The extension of all these assessment requirements with the necessary adjustments to the local contexts leads to a number of clinical trials which is often important. On the contrary, methodological rigor should lead to include a maximum of patients in a minimum of clinical trials in order to maximize the power and furthermore the efficiency of the clinical development.

\section{Proposals for recommendation}

\subsection{Comparison remains the basis of any evaluation}

Clinical trials versus placebo and/or active comparators are necessary, except in certain rare pathologies. In the latter case of historical comparisons are possible if justified and sufficiently referenced. The absence of a control group may be accepted if the methodology allows demonstrating an expected and adequately justified major clinical effect (in particular in terms of survival or on other objective and clinically relevant efficacy criteria such as relapse-free survival or recovery).

\subsection{The use of a non-drug comparator may, or even must, be applied as soon as such a comparator exists and is recognized as a reference by the relevant guidelines}

This type of comparison does not dispense from an evaluation versus placebo of the study drug (except for ethical limits). During the use of a non-drug comparator, the robustness of the results may be affected by the difficulties in performing a double blind under adequate conditions. Furthermore, the infrastructures required for the administration of two types of treatment may vary widely and result in problems in the extrapolation of results from one study to the general population. Nonetheless, this type of comparison may be of interest in the elaboration of therapeutic strategies or to support public health decisions (e.g. appendectomy versus antibiotic therapy). ${ }^{[17]}$

\subsection{The universal comparator that allows answering all the clinical assessment questions does not exist}

Therefore, its choice will be best determined by the target objectives (pharmacological, therapeutic, economic, health policy) but also by the evaluation time (pre- or post- MA), the context (in particular medical or cultural), comparison method, conditions of use of the comparator, type of benefit evaluated or epidemiological context (age of populations, chronic diseases).

\subsection{For the MA, comparison versus placebo remains essential (when possible without loss of opportunity for the patient)}

On the other hand, the use of an active reference comparator is even more desirable when the therapeutic class to which the study drug belongs is extensive.

The need of a study versus active comparator is proportional to the age of the class, and in a class with several representatives, a newcomer should ideally be compared to the previous most effective product in the target indication. Within the framework of the MA, comparison with products with the same mechanism of action will be sought.

\subsection{In terms of ASMR - means "amélioration du service médical rendu" - (clinical added value) assessment, the quantification of the effect can only be carried out in comparison with the current reference drug treatment, or in its absence with respect to the current therapeutic strategy}

The comparator is to be chosen as a function of clinical practice guidelines (see below), which highlights the importance of international, or European at least, harmonization of professional practice recommendations. The conditions of use (posology, duration, etc.) of the comparator must be those that allow the maximum expression of its efficacy within the framework of the guideline (MA, etc.)

\subsection{The use of available guidelines is essential for the choice of the comparator}

The choices as a function of existing guidelines are described in figure 1.

\subsection{The implementation of methodologies that allow demonstrating a superiority on secondary criteria in a non-inferiority study on the main criterion would be desirable}

In a study comparing two active products a non-inferiority may be observed in terms of efficacy on the main criterion and a superiority on other measured criteria (e.g. clinical safety, quality of life). The use of this type of methodology requires that the secondary criteria must be clearly pre-defined in the protocol and cannot be chosen later according to the results obtained. 


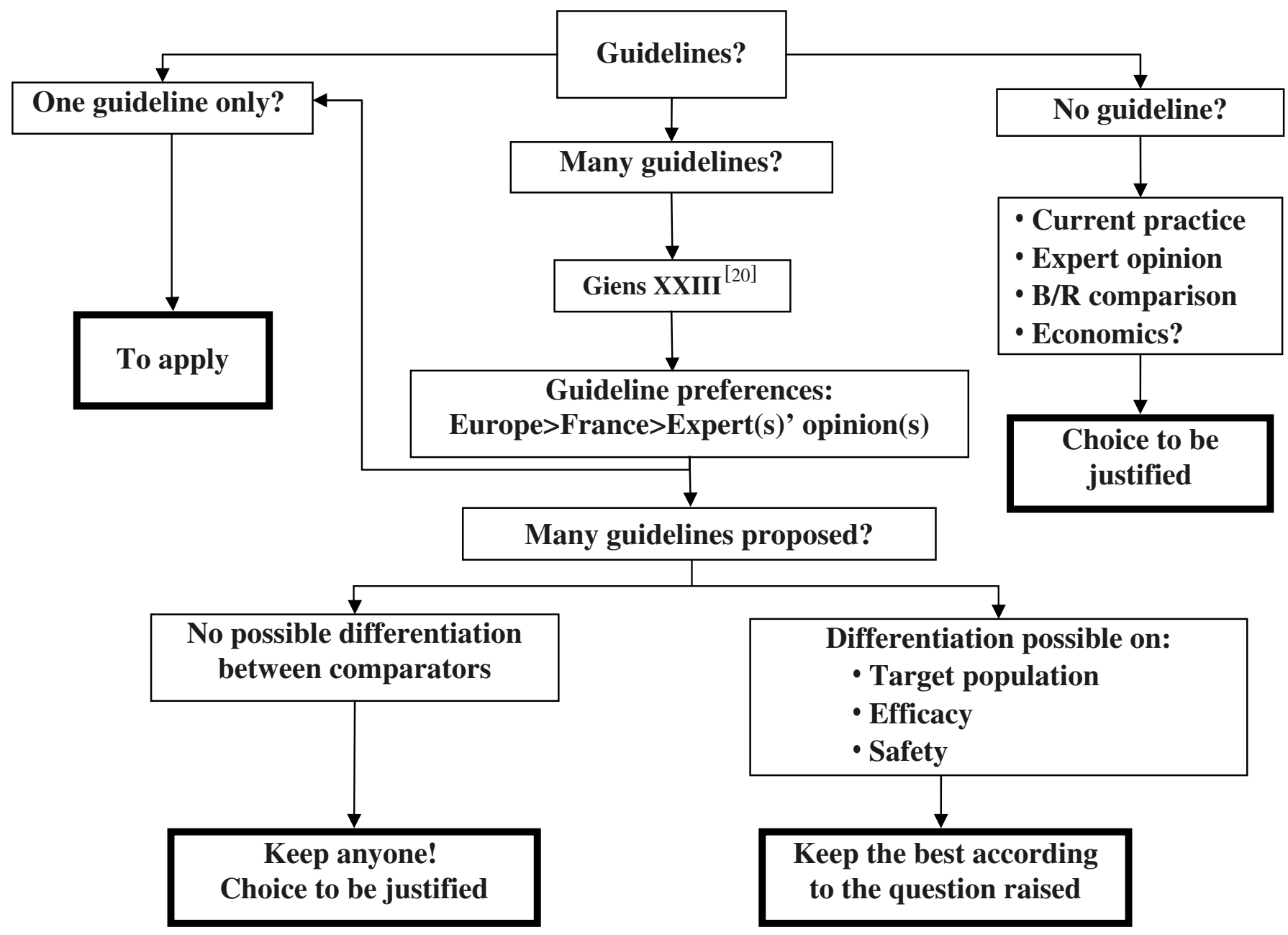

Fig. 1. Use of guidelines for the choice of comparators. B/R: benefit/risk

3.8. Development at the level of the HAS (French Health Authorityl of early end of phase II consultations, comparable to scientific advice provided by the EMA and the Afssaps (French Health Products Safety), to provide the opportunity to better adapt phase III development plans

A part from a presentation of the development plan envisaged, the choice of comparators, administration modes (posologies, dose increase rate, etc.), size of effect expected, target populations and study extrapolation conditions could also be discussed. The implementation of such early consultations (without commitment from the authorities for future opinions according to trials' results) seems even more necessary as they are already common practice among several Health Technology Assessment institutions, in particular the National Institute for Health and Clinical Excellence $^{[18]}$ (NICE) and the Canadian Agency for Drugs and Technology in Health (CADTH). ${ }^{[19]}$
3.9. Considering, at a national and/or European level, the choice of the comparator in the analysis of the specific relationships between the size of effect observed and the expected clinical benefit

The drafting of international recommendations applicable to clinical development, in particular via the ICH process, or at the European level [European Medicines Agency Efficacy Working Party (EMA EWP) guidelines] has contributed to the harmonization of drug clinical development methodology and to increase the robustness of the results observed. As a continuation of this effort and in order to reinforce the pertinence of these results and the efficiency of means employed for this evaluation, it would be advisable to enrich these recommendations with information concerning the choice of comparators and the minimum size of effects that allow confirming a relevant clinical benefit. 


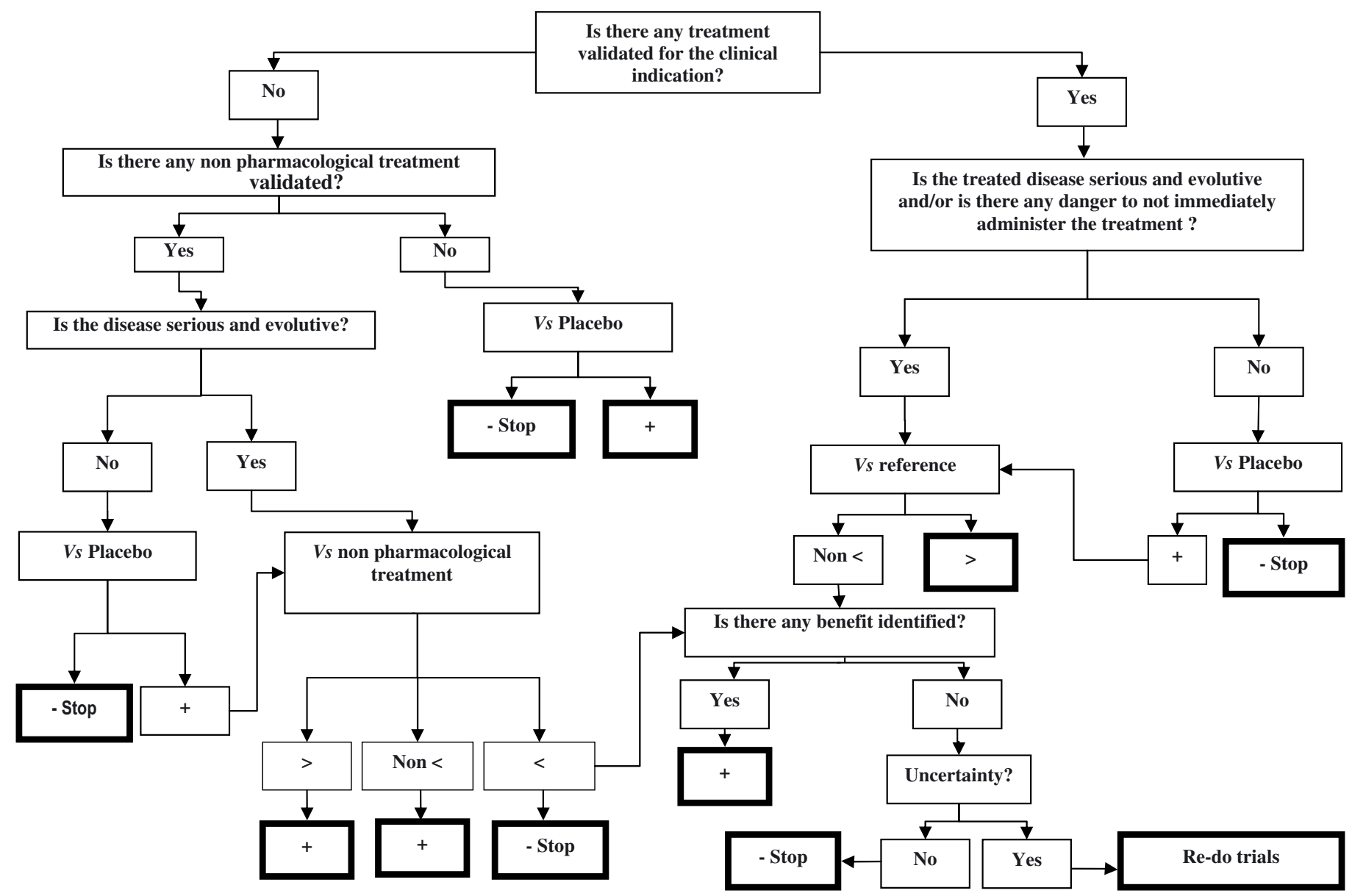

Fig. 2. Use of comparators during clinical development of medicinal products.

\section{Conclusion}

The choice of comparators during the clinical evaluation of medicinal products remains difficult given that as a general rule the comparative data obtained from phase III studies do not always answer the multiple questions. In this context, a comparison with placebo remains a compulsory step (apart from appropriate justification). A direct comparison with an active product, if available, remains the only robust method to demonstrate the clinical added value of a new evaluated drug. The choice of active comparators, whether medicinal or non-medicinal, is based on the careful use of validated guidelines. The development procedure and the choice of comparators are described in figure 2. The use of appropriate statistical methodology combining the demonstration of non-inferiority on a criterion (for example efficacy) and superiority on another (for example safety and quality of life) would allow obtaining more information from the comparative studies performed in this manner. Finally, exchanges at the end of phase
II with the competent authorities would allow a better anticipation of their expectations and thus improve the phase III development plans.

Participants. Alexandre Barna (Direction Générale de la Santé, Paris), Laurent Becquemont (AP-HP, Paris), JeanFrançois Bergman (AP-HP, Paris), Corine Bernaud (Laboratoire Astra-Zeneca), Isabelle Boucot (Laboratoire GlaxoSmithKline), Isabelle Cheiney (Direction de la Sécurité Sociale, Paris), Michel Cucherat (Hôpital Laënnec, Lyon), Anne d'Andon (Haute Autorité de Santé, Saint-Denis la Plaine), Jérôme d'Enfert (Laboratoire Roche), Alain Francillon (Laboratoire Novartis), Elisabeth Frauger (CIC, UPCET, AP-HM, Marseille), Danièle Girault (Laboratoire Wyeth), Jean-Marc Harlin (Mutualité Sociale Agricole), Alain Joseph (Laboratoire Pierre Fabre), Catherine Lassale (LEEM-ARIIS, Paris), Florence Mahlberg-Gaudin (Laboratoire Servier), Thierry Moreau-Defarges (Cyclamed, Boulogne), Mira Pavlovic (Afssaps, Saint-Denis), Sophie Ravoire (Laboratoire Lilly), 
Lise Rochaix (Haute Autorité de Santé, Paris), Eric Vicaut (AP-HP, Paris).

\section{References}

1. EU standard of medicinal product registration: clinical evaluation of risk/benefit - The role of comparator studies. Ref. EMEA/119319/04, October 2004 http: //wWw . ema. europa. eu

2. Van Luijn JCF, Gribnau FWJ, Leufkens HGM. Availability of comparative trials for the assessment of new medicines in the European Union at the moment of market authorization. Br J Clin Pharmacol 2006; 63: 159-62

3. Chalkidou K, Tunis S, Lopert R, et al. Comparative effectiveness research and evidence-based health policy: experience from four countries. The Milbank Quarterly 2009; 87: 339-67

4. Clement FM, Harris A, Li JJ, et al. Using effectiveness and cost-effectiveness to make drug coverage decisions: a comparison of Britain, Australia, and Canada. JAMA 2009; 302: 1437-43

5. Marley J. Efficacy, effectiveness, efficiency. Aust Prescr 2000; 23: 114-5

6. Chalmers I, Glasziou P. Avoidable waste in the production and reporting of research evidence. Lancet 2009; 374: 86-9

7. Atkins D. Creating and synthesizing evidence with decision makers in mind. Med Care 2007; 45: S16-S22

8. Mitka M. Studies comparing treatments ramp up. JAMA 2009; 301: 1975

9. Berwick DM. The science of improvement. JAMA 2008; 299: 1182-4

10. Dawson L, Zarin DA, Emanuel EJ, et al. Considering usual medical care in clinical trial design. PLoS Med 2009; 6: 1-6

11. Rawlins M. Harveian Oration - De testimonio: on the evidence for decisions about the use of therapeutic interventions. Lancet 2008; 372: 2152-61
12. ICH topic E10. Choice of control group in clinical trials. Ref CPMP/ICH/364/96, January 2001 http://www.ema.europa.eu

13. Glickman SW, McHutchinson JG, Peterson ED, et al. Ethical and scientific implications of the globalisation of clinical research. N Eng J Med 2009; 360: $816-23$

14. Luce BR, Kramer JM, Goodman SN, et al. Rethinking randomized clinical trials for comparative effectiveness research: the need for transformational change. Ann Intern Med 2009; 151: 206-9

15. EMEA/CPMP position statement on the use of placebo in clinical trials with regard to the revised declaration of Helsinki. Ref. EMEA/17424/01, June 2001 http://www. ema. europa.eu

16. Garattini S, Chalmers I. Patients and the public deserve big changes in evaluation of drugs. BMJ 2009; 338: 804-6

17. Andersson RE, Petzold MG. Nonsurgical treatment of appendiceal abscess or phlegmon: a systematic review and meta-analysis. Ann Surg 2007; 246: $741-8$

18. http://www.nice.org.uk/aboutnice/scientificadvice/ AboutScientificAdvice.jsp

19. http://www.cadth.ca/index.php/en/cdr/filing-submission/ pre-submission-meetings

20. Table Ronde $\mathbf{n}^{\circ} 2$ de Giens XXIII. Le bon usage du médicament : définition, référentiels, périmètre et champ d'application. Therapie 2008; 63: 267-73

Correspondence and offprints: Driss Berdä̈, Service de Pharmacologie Clinique, CHU de Bordeaux, Zone Nord Carreire, Bâtiment 1A, Place Amélie-Raba-Léon, 33076 Bordeaux Cedex, France.

E-mail: driss.berdai@u-bordeaux2.fr 